\title{
AUTHOR INDEX \\ VOLUME 7 (1993)
}

Adamson, S., see Astley, S.

7 (1993) 1313-1338

Antony, R. T., see Llinas, J.

7 (1993) 285-308

Arcelli, C., Sanniti di Baja, G. \& Kwok, P. C. K., Parallel

Pattern Compression by Octagonal Propagation

7 (1993) 1077-1102

Arkin, R. C., Carter, W. M. \& MacKenzie, D. C., Active

Avoidance: Escape and Dodging Behaviors for Reactive Control

Armstrong, J., see Astley, S.

7 (1993) 175-192

7 (1993) 1313-1338

Arumugam, A., Radhakrishnan, T., Suen, C. Y. \& Wang,

P. S. P., A Thinning Algorithm Based on the Force Between

Charged Particles

Astley, S., see Bowyer, K. W.

7 (1993) 987-1008

7 (1993) 1309-1312

Astley, S., see Miller, P.

Astley, S., Hutt, I., Miller, P., Rose, P., Taylor, C., Boggis, C., Adamson, S., Valentine, T., Davies, J. \& Armstrong, J.,

Automation in Mammography: Computer Vision and Human Perception

Austin, S., see Zavaliagkos, G.

Ayyadurai, S., see Gupta, A.

Barman, H., Granlund, G. \& Haglund, L., Feature Extraction for Computer-Aided Analysis of Mammograms

Barrière, C., see Plamondon, R.

Basak, J., Chaudhury, S., Pal, S. K. \& Majumder, D. D.,

Matching of Structural Shape Descriptions with Hopfield Net

Ben, J. I., see Burges, C. J. C.

Ben-Hanan, U., see Peleg, K.

Bengio, Y., A Connectionist Approach to Speech Recognition

Bentz, J. W., see Bromley, J.

Bhatt, P. C. P., see Saxena, M. K.

Bhatt, P. C. P., see Saxena, M. K.

7 (1993) 1461-1476

Biswas, K. K., see Saxena, M. K.

Bobet, P., see Crowley, J. L.

Bocchieri, E., see Levin, E.

Boggis, C., see Astley, S.

Booker, L. B., see Musman, S. A.

Bottou, L., see Bromley, J.

Bourbakis, N. G., Searching, Reasoning, Constraints Satisfaction for Problem Solving

Bourdeau, M., see Plamondon, R.

Bourlard, H., see Morgan, N.

Bowyer, K. W. \& Astley, S., State of the Art in Digital

Mammographic Image Analysis

Bowyer, K. W., see Christensen, H. I.

7 (1993) 1313-1338

7 (1993) 949-963

7 (1993) 757-773

7 (1993) 1339-1356

7 (1993) 1247-1270

7 (1993) 377-404

7 (1993) 689-704

7 (1993) 917-933

7 (1993) 647-667

7 (1993) 669-688

7 (1993) 193-196

7 (1993) 221-242

7 (1993) 221-242

7 (1993) 109-122

7 (1993) 783-799

7 (1993) 1313-1338

7 (1993) 513-526

7 (1993) 669-688

7 (1993) 407

7 (1993) 1247-1270

7 (1993) 899-916

7 (1993) 1309-1312

7 (1993) 1-8

1507 
Bowyer, K. W., see Woods, K. S.

7 (1993) 1417-1436

Bowyer, K. W., see Stark, L.

7 (1993) 573-594

Bromley, J., Bentz, J. W., Bottou, L., Guyon, I., LeCun, Y., Moore, C., Säckinger, E. \& Shah, R., Signature Verification Using A "Siamese" Time Delay Neural Network

7 (1993) 669-688

Bruynooghe, M., Recent Results in Hierarchical Clustering. I - The Reducible Neighborhoods Clustering Algorithm

Brzakovic, D. \& Neskovic, M., Mammogram Screening Using Multiresolution-Based Image Segmentation

Bunke, H., see Christensen, H. I.

Burges, C. J. C., Ben, J. I., Denker, J. S., LeCun, Y. \& Nohl, C. R., Off Line Recognition of Handwritten Postal Words Using Neural Networks

Carter, W. M., see Arkin, R. C.

Chang, L. W., see Musman, S. A.

Chaudhuri, B. B., see Sengupta, P.

Chaudhury, S., see Basak, J.

Chen, C. \& Trivedi, M. M., SAVIC: A Simulation, Visualization and Interactive Control Environment for Mobile Robots

Chen, J., see Lee, S.

Chen, J.-S. \& Srihari, S. N., Hypothesis Generation in Conflict-Based Diagnosis

Chin, R. T., see Jang, B. K.

Chitre, Y., Dhawan, A. P. \& Moskowitz, M., Artificial Neural Network Based Classification of Mammographic Microcalcifications Using Image Structure Features

Christensen, H. I., A Low-Cost Robot Camera Head

Christensen, H. I., Bowyer, K. W. \& Bunke, H., Active Robot Vision: Camera Heads, Model Based Navigation and Reactive Control

Clark, J. J., see Ferrier, N. J.

Close, R., see Kim, E.-K.

Cohen, M., see Morgan, N.

Craig, I. D., Formal Techniques in the Development of Blackboard Systems

Crowley, J. L., Bobet, P. \& Mesrabi, M., Layered Control of a Binocular Camera Head

Davies, J., see Astley, S.

Decker, K., Garvey, A., Humphrey, M. \& Lesser, V., Control Heuristics for Scheduling in a Parallel Blackboard System

Decker, K., Garvey, A., Humphrey, M. \& Lesser, V., A RealTime Control Architecture for an Approximate Processing Blackboard System

Denker, J. S., see Burges, C. J. C.

Desautels, J. E. L., see Shen, L.

Dhawan, A. P., see Chitre, Y.

Dimauro, G., Impedovo, S. \& Pirlo, G., A New Thinning Algorithm Based on Controlled Deletion of Edge Regions

Doss, C. C., see Woods, K. S.

$7(1993) 689-704$
$7(1993) 175-192$
$7(1993) 513-526$
$7(1993) 595-619$
$7(1993) 377-404$

7 (1993) 123-144

7 (1993) 493-512

7 (1993) $475-492$

7 (1993) 1145-1181

7 (1993) 1377-1401

7 (1993) 69-87

7 (1993) 1-8

7 (1993) 9-31

7 (1993) 775-782

7 (1993) 899-916

7 (1993) 197-219

7 (1993) $109-122$

7 (1993) 1313-1338

7 (1993) 243-264

7 (1993) 265-284

7 (1993) 689-704

7 (1993) 1403-1416

7 (1993) 1377-1401

7 (1993) 969-986

7 (1993) 1417-1436 
Draper, B. A., Hanson, A. R. \& Riseman, E. M., Learning Blackboard-Based Scheduling Algorithms for Computer Vision

7 (1993) 309-328

Drucker, H., Schapire, R. \& Simard, P., Boosting Performance in Neural Networks

Eckhardt, U. \& Maderlechner, G., Invariant Thinning

Eklundh, J.-O., see Pahlavan, K.

Enguehard, C., Trigano, P. \& Malvache, P., ANA: Automatic Natural Acquisition

Ferrier, N. J. \& Clark, J. J., The Harvard Binocular Head

Fogelman Soulie, F., Viennet, E. \& Lamy, B., Multi-Modular Neural Network Architectures: Applications in Optical Character and Human Face Recognition

Franco, H., see Morgan, N.

Garvey, A., see Decker, K.

Garvey, A., see Decker, K.

Giles, C. L., see Miller, C. B.

Gorin, A. L., see Miller, L. G.

Granlund, G., see Barman, H.

Gupta, A., Nagendraprasad, M. V., Liu, A., Wang, P. S. P. \& Ayyadurai, S., An Integrated Architecture for Recognition of Totally Unconstrained Handwritten Numerals

Guyon, I. \& Wang, P. S. P., Advances in Pattern Recognition Systems Using Neural Network Technologies

Guyon, I., see Bromley, J.

Haglund, L., see Barman, $H$.

Hall, L. O., see Stark, L.

Hanson, A. R., see Draper, B. A.

Hau, H.-Y., Decomposition of Belief Function in Hierarchical Hypotheses Space

Hong, J., see Liu, W.

Hsieh, C.-C. \& Lee, H.-J., A Probabilistic Stroke-Based Viterbi Algorithm for Handwritten Chinese Characters Recognition

Hu, G. \& Li, Z.-N., An X-Crossing Preserving Skeletonization Algorithm

Hughes, J. G., see Liu, W.

Humphrey, M., see Decker, K.

Humphrey, M., see Decker, K.

Hutt, I., see Astley, S.

Impedovo, S., see Dimauro, G.

Inoue, M., see Kim, E.-K.

Israel, P., see Koutsougeras, C.

Jain, R., see Roth, Y.

Jang, B. K. \& Chin, R. T., Reconstructable Parallel Thinning

Jenkin, M., see Milios, E.

Karssemeijer, N., Adaptive Noise Equalization and Recognition of Microcalcification Clusters in Mammograms

Kawai, H., see Kim, E.-K.

Kibler, D., see Ruby, D.

Kim, E.-K., Wu, J.-T., Tamura, S., Sato, Y., Close, R.,

Taketani, H., Kawai, H., Inoue, M. \& Ono, K. Comparison 
of Neural Network and K-NN Classification Methods in Vowel and Patellar Subluxation Image Recognitions

Koutsougeras, C. \& Israel, P., A Method for Parallel Search

Under Higher-Order Constraints

Kwok, P. C. K., see Arcelli, C.

Lam, L. \& Suen, C. Y., Automatic Comparison of Skeletons by Shape Matching Methods

Lam, L., see Lee, S.-W.

Lamy, B., see Fogelman Soulie, F.

LeCun, Y., see Bromley, J.

LeCun, Y., see Burges, C. J. C.

Lee, H.-J., see Hsieh, C.-C.

Lee, S. \& Chen, J., Building Models for Qualitative Prediction of System Dynamic Behavior

Lee, S.-W., Lam, L. \& Suen, C. Y., A Systematic Evaluation of Skeletonization Algorithms

Lesser, V., see Decker, K.

Lesser, V., see Decker, K.

Levin, E., Pieraccini, R. \& Bocchieri, E., Time-Warping Network: A Neural Approach to Hidden Markov Model Based Speech Recognition

Li, B., see Zhao, Q.

Li, H. \& Wang, J., Computing Optical Flow with a Recurrent Neural Network

Li, W. \& Nasrabadi, N. M., Invariant Object Recognition

Based on a Neural Network of Cascaded RCE Nets

$\mathrm{Li}, \mathrm{Z} . \mathrm{-N}$., see $\mathrm{Hu}$, G.

Liu, A., see Gupta, A.,

Liu, R.-L. \& Soo, V.-W., Parsing-Driven Generalization for Natural Language Acquisition

Liu, W., Hong, J., McTear, M. F. \& Hughes, J. G., An Extended Framework for Evidential Reasoning Systems

Llinas, J. \& Antony, R. T., Blackboard Concepts for Data Fusion Applications

MacKenzie, D. C., see Arkin, R. C.

Maderlechner, G., see Eckhardt, U.

Majumder, D. D., see Basak, J.

Makhoul, J., see Zavaliagkos, G.

Malvache, P., see Enguehard, C.

Martin, G. L., Rashid, M. \& Pittman, J. A., Integrated

Segmentation and Recognition Through Exhaustive

Scans or Learned Saccadic Jumps

McTear, M. F., see Liu, W.

Mesrabi, M., see Crowley, J. L.

Milios, E., Jenkin, M. \& Tsotsos, J., Design and Performance of TRISH, a Binocular Robot Head with Torsional

Eye Movements

Miller, C. B. \& Giles, C. L., Experimental Comparison of the Effect of Order in Recurrent Neural Networks

Miller, L. G. \& Gorin, A. L., Structured Networks for Adaptive Language Acquisition

7 (1993) 775-782

7 (1993) 409-429

7 (1993) 1077-1102

7 (1993) 1271-1286

7 (1993) 1203-1225

7 (1993) 721-755

7 (1993) 669-688

7 (1993) 689-704

7 (1993) 329-352

7 (1993) 493-512

7 (1993) 1203-1225

7 (1993) 243-264

7 (1993) 265-284

7 (1993) 783-799

7 (1993) 431-440

7 (1993) 801-814

7 (1993) 815-829

7 (1993) 1031-1053

7 (1993) 757-773

7 (1993) 621-644

7 (1993) 441-457

7 (1993) 285-308

7 (1993) 175-192

7 (1993) 1115-1144

7 (1993) 377-404

7 (1993) 949-963

7 (1993) 353-375

7 (1993) 831-847

7 (1993) 441-457

7 (1993) 109-122

7 (1993) 51-68

7 (1993) 849-872

7 (1993) 873-898 
Miller, P. \& Astley, S., Automated Detection of Mammographic Asymmetry Using Anatomical Features

7 (1993) 1461-1476

Miller, P., see Astley, S.

Moore, C., see Bromley, J.

Morgan, N., Bourlard, H., Renals, S., Cohen, M. \&

Franco, H., Hybrid Neural Network/Hidden Markov Model Systems for Continuous Speech Recognition

Mori, S., see Suzuki, T.

Moskowitz, M., see Chitre, Y.

Musman, S. A., Chang, L. W. \& Booker, L. B., Application of a Real-Time Control Strategy for Bayesian Belief

Networks to Ship Classification Problem Solving

7 (1993) 1313-1338

7 (1993) 669-688

Nagendraprasad, M. V., see Gupta, A.

Nasrabadi, N. M., see Li, W.

Neskovic, M., see Brzakovic, D.

Nohl, C. R., see Burges, C.J.C.

Ono, K., see Kim, E.-K.

Pahlavan, K. \& Eklundh, J.-O., Head, Eyes and Head-Eye Systems

Pal, S. K., see Basak, J.

Parker, G. A., see Pretlove, J.R.G.

Peleg, K. \& Ben-Hanan, U., Adaptive Classification by Neural

Net Based Prototype Populations

Philip Kegelmeyer, W., Jr., see Woods, K. S.

Philip Kegelmeyer, W., Jr., Evaluation of Stellate Lesion

Detection in a Standard Mammogram Data Set

Pieraccini, R., see Levin, E.

Pirlo, G., see Dimauro, G.

Pisano, E. D. \& Shtern, F., Image Processing and Computer Aided Diagnosis in Digital Mammography: A Clinical Perspective

Pittman, J. A., see Martin, G. L.

Plamondon, R., Suen, C. Y., Bourdeau, M. \& Barrière, C., Methodologies for Evaluating Thinning Algorithms for Character Recognition

Poty, V. \& Ubeda, S., A Parallel Thinning Algorithm Using $\mathrm{K} x \mathrm{~K}$ Masks

Pretlove, J. R. G. \& Parker, G. A., The Surrey Attentive Robot Vision System

Priebe, C. E., see Woods, K.S.

Radhakrishnan, T., see Arumugam, A.

Rangayyan, R. M., see Shen, L.

Rashid, M., see Martin, G. L.

Renals, S., see Morgan, N.

Riseman, E. M., see Draper, B. A.

Rose, P., see Astley, S.

Roth, Y. \& Jain, R., Simulation and Expectation in SensorBased Systems

Ruby, D. \& Kibler, D., Learning Steppingstones for Problem Solving

Säckinger, E., see Bromley, J.

Sanniti di Baja, G., see Arcelli, C.

Sato, Y., see Kim, E.-K.

7 (1993) 899-916

7 (1993) 1055-1076

7 (1993) 1377-1401

7 (1993) 513-526

7 (1993) 757-773

7 (1993) 815-829

7 (1993) 1437-1460

7 (1993) 689-704

7 (1993) 775-782

7 (1993) 33-49

7 (1993) 377-404

7 (1993) 89-107

7 (1993) 917-933

7 (1993) 1417-1436

7 (1993) 1477-1492

7 (1993) 783-799

7 (1993) 969-986

7 (1993) 1493-1502

7 (1993) 831-847

7 (1993) 1247-1270

7 (1993) 1183-1202

7 (1993) 89-107

7 (1993) 1417-1436

7 (1993) 987-1008

7 (1993) 1403-1416

7 (1993) 831-847

7 (1993) 899-916

7 (1993) 309-328

7 (1993) 1313-1338

7 (1993) 145-173

7 (1993) 527-540

7 (1993) 669-688

7 (1993) 1077-1102

7 (1993) 775-782 
Saxena, M. K. \& Bhatt, P. C. P., Blackboard Systems

Saxena, M. K., Biswas, K. K. \& Bhatt, P. C. P., DKRL - An

Object-Oriented Language to Support Problem Solving in

Distributed Blackboard Systems

Schapire, R., see Drucker, H.

Schwartz, R., see Zavaliagkos, G.

Sengupta, P. \& Chaudhuri, B. B., A Morpho-Syntactic Analysis Based Lexical Subsystem

Shah, R., see Bromley, J.

Shen, L., Rangayyan, R. M. \& Desautels, J. E. L., Detection and Classification of Mammographic Calcifications

Shtern, F., see Pisano, E. D.

Simard, P., see Drucker, H.

Sklansky, J., see Suzuki, S.

Solka, J. L., see Woods, K.S.

Soo, V.-W., see Liu, R.-L.

Srihari, S. N., see Chen, J.-S.

Stark, L., Hall, L. O. \& Bowyer, K. W., Methods for Combination of Evidence in Function-Based 3-D Object Recognition

Suen, C. Y. \& Wang, P. S. P., Thinning Methodologies for Pattern Recognition

Suen, C. Y., see Lee, S.-W.

Suen, C. Y., see Plamondon, R.

Suen, C. Y., see Lam, L.

Suen, C. Y., see Arumugam, A.

Suzuki, S., Ueda, N. \& Sklansky, J., Graph-Based Thinning for Binary Images

Suzuki, T. \& Mori, S., Structural Description of Line Images by the Cross Section Sequence Graph

Taketani, H., see Kim, E.-K.

Tamura, S., see Kim, E.-K.

Taylor, C., see Astley, S.

Trigano, P., see Enguehard, C.

Trivedi, M. M., see Chen, C.

Tsotsos, J., see Milios, E.

Ubeda, S., A Parallel Thinning Algorithm Using the Bounding Boxes Techniques

Ubeda, S., see Poty, V.

Ueda, N., see Suzuki, S.

Valentine, T., see Astley, S.

van Vliet, L. J., see Verwer, B. J. H.

Verbeek, P. W., see Verwer, B. J. H.

Verwer, B. J. H., van Vliet, L. J. \& Verbeek, P. W., Binary

and Grey-Value Skeletons: Metrics and Algorithms

Viennet, E., see Fogelman Soulie, F.

von der Malsburg, C., see Wiskott, L.

Wang, J., see Li, H.

Wang, P. S. P., see Zhang, Y. Y.

Wang, P. S. P., see Guyon, I.

Wang, P. S. P., see Gupta, A.

Wang, P. S. P., see Suen, C. Y.
7 (1993) 193-196

7 (1993) 221-242

7 (1993) 705-719

7 (1993) 949-963

7 (1993) 595-619

7 (1993) 669-688

7 (1993) 1403-1416

7 (1993) 1493-1502

7 (1993) 705-719

7 (1993) 1009-1030

7 (1993) 1417-1436

7 (1993) 621-644

7 (1993) 475-492

7 (1993) 573-594

7 (1993) 965-967

7 (1993) 1203-1225

7 (1993) 1247-1270

7 (1993) 1271-1286

7 (1993) 987-1008

7 (1993) 1009-1030

7 (1993) 1055-1076

7 (1993) 775-782

7 (1993) 775-782

7 (1993) 1313-1338

7 (1993) 353-375

7 (1993) 123-144

7 (1993) 51-68

7 (1993) 1103-1114

7 (1993) 1183-1202

7 (1993) 1009-1030

7 (1993) 1313-1338

7 (1993) 1287-1308

7 (1993) 1287-1308

7 (1993) 1287-1308

7 (1993) 721-755

7 (1993) 935-948

7 (1993) 801-814

7 (1993) 1227-1246

7 (1993) 645-646

7 (1993) 757-773

7 (1993) 965-967 
Wang, P. S. P., see Arumugam, A.

7 (1993) 987-1008

Wiskott, L. \& von der Malsburg, C., A Neural System for the Recognition of Partially Occluded Objects in Cluttered Scenes: A Pilot Study

Woods, K. S., Doss, C. C., Bowyer, K. W., Solka, J. L., Priebe, C. E. \& Philip Kegelmeyer, W.,Jr., Comparative Evaluation of Pattern Recognition Techniques for Detection of Microcalcifications in Mammography

$7(1993) 1417-1436$

Wu, J.-T., see Kim, E.-K.

Zavaliagkos, G., Austin, S., Makhoul, J. \& Schwartz, R., A Hybrid Continuous Speech Recognition System Using Segmental Neural Nets with Hidden Markov Models

Zhang, Y. Y. \& Wang, P. S. P., Analytical Comparison of Thinning Algorithms

Zhao, Q. \& Li, B., M: An Approximate Reasoning System

7 (1993) $775-782$

7 (1993) 949-963

7 (1993) $1227-1246$

7 (1993) 431-440 